\title{
Inverse Compton Backscattering Source driven by the multi 10 TW-Laser installed at Daresbury
}

\author{
G. PRIEBE,1 D. LAUNDY,1 M.A. MACDONALD,1 G.P. DIAKUN,1 S.P. JAMISON,3 \\ P.J. PHILLIPS,4 B. SHEEHY,9 L.B. JONES,1,3 D.J. HOLDER,1,3 S. L. SMITH,1,2 G.J. \\ HIRST,5 \\ N. NAUMOVA,11 G.A. KRAFFT,13 K. SPOHR,12 H. LEGALL,10 J.B. ROSENZWEIG,14 \\ F. GRÜNER,8 C.P.J. BARTY,6 C. SIDERS,6 U. SCHRAMM,7 S. CHATTOPADHYAY 2 \\ AND
}

E.A. SEDDON1

1Science and Technology Facilities Council, Daresbury Laboratory, Cheshire, WA4 4AD, UK 2Cockcroft Institute, Daresbury Science and Innovation Campus, Warrington, WA4 4AD, UK 3Accelerator Science and Technology Centre, Daresbury Laboratory, Cheshire, WA4 4AD, UK ${ }_{4}$ University of Dundee, Division of Electronic Engineering and Physics, Dundee, DD1 4HN, UK 5STFC Rutherford Appleton Laboratory, Chilton, Didcot, OX11 0QX, UK 6Lawrence Livermore National Laboratory, Livermore, CA 94551, USA 7Forschungszentrum Dresden-Rossendorf, 01328 Dresden, Germany 8Max-Planck-Institut für Quantenoptik, 85748 Garching, Germany 9Brookhaven National Laboratory,Upton, NY 11973-5000, USA 10Max Born Institut, Max Born Str. 2a, D-12489 Berlin, Germany 11Laboratoire d'Optique Appliquee, Chemin de la Huniere, 91761 Palaiseau, France ${ }_{12}$ Department of Electronic Engineering and Physics, University of Paisley, Glasgow, PA1 2BE, UK 13Thomas Jefferson National Accelerator Facility, Jefferson Avenue, Newport News, VA 23606, USA ${ }_{14}$ University of California at Los Angeles, Department of Physics and Astronomy, Los Angeles, CA, USA 


\title{
Inverse Compton Backscattering Source driven by the multi 10 TW-Laser installed at Daresbury*
}

\author{
G. PRIEBE, ${ }^{1} \quad$ D. LAUNDY, ${ }^{1}$ M.A. MACDONALD, ${ }^{1}$ G.P. DIAKUN, ${ }^{1} \quad$ S.P. JAMISON,${ }^{3}$ \\ P.J. PHILLIPS, ${ }^{4}$ B. SHEEHY, ${ }^{9}$ L.B. JONES, ${ }^{1,3}$ D.J. HOLDER, ${ }^{1,3}$ S. L. SMITH, ${ }^{1,2}$ G.J. HIRST, ${ }^{5}$ \\ N. NAUMOVA, ${ }^{11}$ G.A. KRAFFT, ${ }^{13}$ K. SPOHR, ${ }^{12}$ H. LEGALL, ${ }^{10}$ J.B. ROSENZWEIG, ${ }^{14}$ \\ F. GRÜNER,${ }^{8}$ C.P.J. BARTY, ${ }^{6}$ C. SIDERS,${ }^{6}$ U. SCHRAMM, ${ }^{7}$ S. CHATTOPADHYAY ${ }^{2}$ AND \\ E.A. SEDDON ${ }^{1}$
}

${ }^{1}$ Science and Technology Facilities Council, Daresbury Laboratory, Cheshire, WA4 4AD, UK

${ }^{2}$ Cockcroft Institute, Daresbury Science and Innovation Campus, Warrington, WA4 4AD, UK

${ }^{3}$ Accelerator Science and Technology Centre, Daresbury Laboratory, Cheshire, WA4 4AD, UK

${ }^{4}$ University of Dundee, Division of Electronic Engineering and Physics, Dundee, DD1 4HN, UK

${ }^{5}$ STFC Rutherford Appleton Laboratory, Chilton, Didcot, OX11 0QX, UK

${ }^{6}$ Lawrence Livermore National Laboratory, Livermore, CA 94551, USA

${ }^{7}$ Forschungszentrum Dresden-Rossendorf, 01328 Dresden, Germany

${ }^{8}$ Max-Planck-Institut für Quantenoptik, 85748 Garching, Germany

${ }^{9}$ Brookhaven National Laboratory,Upton, NY 11973-5000, USA

${ }^{10}$ Max Born Institut, Max Born Str. 2a, D-12489 Berlin, Germany

${ }^{11}$ Laboratoire d'Optique Appliquee, Chemin de la Huniere, 91761 Palaiseau, France

${ }^{12}$ Department of Electronic Engineering and Physics, University of Paisley, Glasgow, PA1 2BE, UK

${ }^{13}$ Thomas Jefferson National Accelerator Facility, Jefferson Avenue, Newport News, VA 23606, USA

${ }^{14}$ University of California at Los Angeles, Department of Physics and Astronomy, Los Angeles, CA, USA

\begin{abstract}
Inverse Compton scattering is a promising method to implement a high brightness, ultra-short, energy tuneable X-ray source at accelerator facilities. We have developed an inverse Compton backscattering X-ray source driven by the multi $10 \mathrm{TW}$ laser installed at Daresbury. Hard X-rays, with spectral peak ranging from 15 to $30 \mathrm{keV}$, depending on the scattering geometry, will be generated through the interaction of a laser pulse with an electron bunch delivered by the energy recovery linear accelerator prototype at Daresbury. X-ray pulses containing $9 \times 10^{7}$ photons per pulse will be created from head on collisions, with an pulse duration comparable to the incoming electron bunch length. For transverse collisions $8 \times 10^{6}$ photons per pulse will be generated, where the laser pulse transit time defines the X-ray pulse duration. The peak spectral brightness is predicted to be $\sim 10^{21}$ photons $/ \mathrm{s} / \mathrm{mm}^{2} / \mathrm{mrad}^{2} / 0.1 \% \Delta E / E$, which is comparable to fourth generation synchrotron light sources.
\end{abstract}

Keywords: Compton scattering, Thomson scattering, Laser synchrotron radiation, Compton synchrotron radiation, Laser Compton scattering, X-ray source, ultra-short X-ray pulses, energy recovery linac, ERLP.

\section{INTRODUCTION}

Knowledge of the atomic structure of matter is of fundamental importance in science. The discovery of structural information has often led to the creation of new scientific fields. Advances in solid state physics

Address correspondence and reprint requests to: G. Priebe, Science and Technology Facilities Council, Daresbury Laboratory, Cheshire, WA4 4AD, UK. E-mail: g.priebe@dl.ac.uk

*This paper was presented at the SPIE conference Soft X-Ray Lasers and Applications VII in San Diego, USA. followed the discovery of crystal structures obtained through the production of X-ray diffraction patterns (von Laue, 1936). The modern science of molecular biology and genetic engineering were triggered by the discovery of the double helix structure of DNA (Watson \& Crick, 1953). X-ray diffraction give precise information about atomic structure on a sub angstrom scale and spectroscopic techniques - Extended X-ray Absorption Fine Structure (EXAFS), X-ray Absorption Near Edge Structure (XANES) - give information about local order and on chemical states. The possibil- 
ity of generating ultra-short X-ray pulses gives the possibility of observing structural and electronic changes dynamically, for example, observing the detailed intermediate steps of chemical reactions with atomic resolution. Typical timescales for changes in atomic configurations are comparable with the cyclic duration of molecular vibrations. Advances in the generation of ultra-short laser pulses over the past two decades have made it possible to observe these fundamental processes. These advances have taken science from the $n s$ - and $p s$ regime a generation ago, to the $f_{s}$ timescale in the past decade, and recently into the as regime (Strickland \& Mourou, 1985; Sansone et al., 2006; Baker et al., 2006). The fact that the wavelength of these ultra-short laser light sources is significantly larger than the sizes and distances of the constituent atoms in the molecules and crystals under study has meant that information on structural changes could only be inferred from observation of the secondary effects that these structural changes have on optical properties. The recent development of small, affordable, highpower lasers based on chirped pulse amplification (CPA), capable of producing $f s$ pulses has produced an interesting insight into tabletop size X-ray source production. Several $n s$ and $p s \mathrm{X}$-ray techniques based on both synchrotron and table-top X-ray sources have been developed during the past decade (Helliwell \& Rentzepis, 1997). Most of these techniques use an ultrashort laser pulse to initiate a photoreaction in the sample to be studied followed by a delayed ultra-short $\mathrm{X}$-ray pulse which probes the structural changes that take place in the sample. In the solid state, non-thermal melting and laser-induced heat and strain propagation in metal and semiconductor single and mosaic-crystals have been studied by means of time-resolved X-ray diffraction with $n s$ to $p s$ time resolution (Tomov et al., 1999; Siders et al., 1999). The use of all of these techniques in conjunction with an $f s \mathrm{X}$-ray source will allow the direct measurement of structural information in many ultra-fast fundamental physical, chemical and biological processes, currently inaccessible with the time resolution of existing sources. The development of short and ultra-short X-ray sources will drive many important scientific applications by enabling direct measurement of atomic motion and structural changes in the condensed phase. Perhaps the most important feature of these new sources of ultra-short X-ray pulses is that they enable physical experiments combining $f_{S}$ time resolution and atomic scale spatial resolution.

There are several different ways to generate short and ultra-short X-ray pulses (Kim et al., 1994; Arthur et al., 1995; Schoenlein et al., 1996; Schoenlein et al., 2000; Leemans et al., 2000; Catravas et al., 2001; Guo et al., 2001; Service, 2002; Hafz et al., 2003; Phuoc et al., 2003; Umstadter, 2003; Xia al., 2004; Janulweicz et al., 2004; Krafft, 2004; Lucianetti et al. 2004; Giulietti et al., 2005; Tümmler et al. 2005; Zhavoronkov et al., 2005; Janulweicz et al., 2005; Krafft et al., 2005; Riley et al., 2005; Legall et al., 2006; Notley et al., 2006; Khattak et al., 2007; Chen et al., 2008) such as ultrashort laser-generated $\mathrm{K}_{\alpha}$ sources, X-ray free-electron lasers and electron bunch-slicing in synchrotrons, and the one to be discussed here, the inverse Compton scattering of an intense laser pulse from a high-brightness electron beam. Using the laser as an effective undulator with much shorter period than the commonly used magnetic undulators in synchrotron light sources, a much less energetic electron beam can be used. This source also offers a wide tuning range, where X-ray source parameters such as photon energy, brightness, bandwidth, and pulse length can be tuned through the laser and electron beam parameters as well as the interaction geometry of the beams. The interaction can also be viewed as a collision between an electron and photon. This treatment of the system gives rise to Compton scattering or Thomson scattering when the incident photon energy in the electron rest frame is much less than $m c^{2}$. Depending on the reference frame, it can appear as if the electrons have been energized by the photons or if the electrons are moving relativistically, the energy is instead transferred from electrons to photons, therefore the opposite of Compton scattering inverse Compton scattering-. The frequency of the photons scattered off the relativistic electron beam is dependent on the interaction angle at which the two beams meet, typically ranging from $90^{\circ}$ to $180^{\circ}$, although small-angle Thomson scattering has also been a proposed interaction configuration (Huang et al., 1994). The generation of $300 \mathrm{fs}$ hard X-ray pulses has been demonstrated by $90^{\circ}$ Thomson scattering between $f_{s}$ laser pulses and tightly-focused nanosecond electron bunches in a synchrotron (Kim et al., 1994). The relatively low X-ray flux of $\approx 10^{6}$ photons $/ \mathrm{s}$ obtained in these experiments could be significantly increased by the use of higher laser powers and the use of high quality $f s$ electron bunches.

In this paper, we describe a new source of ultrashort X-ray pulses based on inverse Compton scattering of multi terawatt $100 \mathrm{fs}$ laser-pulses with $35 \mathrm{MeV}$ electron bunches delivered by the energy recovery linear accelerator prototype (ERLP) that is being constructed at Daresbury Laboratory (Smith et al., 2005). Theoretically, Thomson scattering from a relativistic electron beam has been extensively studied both in the linear and the nonlinear regime. For clarity, we adapt the linear theory, where the energy of the scattered photon is calculated using energy momentum conservation, and the differential scattering cross 
section is calculated using the theory of quantum electrodynamics. The X-ray source, the inverse COmpton BAckscattering X-ray source driven by the tabletop 10 terawatt ( $\mathrm{T}^{3}$-laser) Laser installed at Daresbury (COBALD) will initially be used as a short pulse diagnostic of the ERLP electron beam (Leemans et al., 1996; Ross et al., 1997; Tannebaum, 1999; Hartemann et al., 2004; Chouffani et al., 2006). It will explore the extreme challenges of photon/electron-beam synchronization which are a fundamental requirement for the next generation of accelerator based light source scientific program. The COBALD source will be exploited for a fast melting experiment (Wark, 1999; Sundaram \& Mazur, 2002; von der Linde, 2003; Sokolowski-Tinten \& von der Linde, 2004) providing information about the synchronization between the laser pulse and the electron bunch, and on the X-ray source stability. In the experiment, a portion of the laser beam will be used to induce disorder at the surface of a crystal which subsequently re-crystallizes on a $p s$ time scale. The X-ray pulse probes the re-crystallization by allowing measurement of the diffracted X-ray intensity from the crystal lattice at times through the transformation. The inverse Compton backscattering scattering source has the desired high-brightness, short pulse, tunable energy research tool, capable of measuring on an atomic scale with high spatial and temporal resolution.

\subsection{ENERGY RECOVERING LINAC}

Synchrotron Radiation (SR) X-ray sources have come a long way from the first parasitic uses of high energy physics machines. Improvements in electron storage rings and insertion devices have led to an exponential increase in brilliance for each successive generation of the sources. The technology of storage rings has reached maturity stage, resulting in $3^{\text {rd }}$ generation SR facilities that are capable of providing a beam quality close to the physical limits. The fundamental X-ray beam properties from ring sources, such as the source size, brilliance and pulse duration are limited by the dynamic equilibrium between the electron beam and the magnet lattice of the storage ring. In contrast to storage rings, linear accelerators can produce both low emittance and very short bunches, because the limitations set by this equilibrium are not an issue. As a result, several proposed large light-source projects are based on linear accelerators. However, in a traditional linear accelerator, the absence of beam recirculation would lead to an enormous power consumption by the electron beam if high average currents are to be obtained. To circumvent this prohibitively large power consumption, Energy Recovery Linac (ERL) tech-nology (Tigner,
1965 ) is being explored for next-generation synchrotron light sources; in such accelerators the energy delivered to the electron beam is re-circulated, rather than the electron beam itself. The use of ERLs produces photon beams with extraordinary brilliance with small source size, with concomitant high transverse coherence and ultra-short pulses. Several laboratories have proposed high-power ERLs for the production of high-brightness radiation. Accelerators for different parameter sets and various applications are being worked on by Cornell University, Argonne National Laboratory, Novosibirsk, KEK and JLAB (Kulipanov et al., 1998; Bazerov et al., 2001; Suwada, et al., 2002; Smith et al., 2004; Neil et al., 2005; Borland, 2005).

An advanced energy recovery linac based light source; a technology demonstrator known as Energy Recovery Linac Prototype (Fig. 1, Tab. 1) is being constructed at Daresbury Laboratory (Poole, et al., 2003; Smith et al., 2005).



Fig. 1. Layout of the Energy Recovery Linac Prototype installed at Daresbury Laboratory.

It is based on a combination of a DC photocathode electron gun, a superconducting injector linac and main linac operating in energy recovery mode. A cavity IR-FEL is incorporated in the machine. It is based on a fixed gap undulator that will deliver intense short pulses of photons in the wavelength range $4 \mu \mathrm{m}$ to $12 \mu \mathrm{m}$. The $1.4 \mathrm{ps}$ pulses

Tab. 1. The main parameters of the energy recovery linear accelerator prototype (ERLP).

\begin{tabular}{|c|c|}
\cline { 2 - 2 } \multicolumn{1}{c|}{} & ERLP \\
\hline gun energy & $350 \mathrm{keV}$ \\
\hline max energy & $35 \mathrm{MeV}$ \\
\hline charge / bunch & $80 \mathrm{pC}$ \\
\hline bunch rep. rate & $81.25 \mathrm{MHz}$ \\
\hline $\begin{array}{c}\text { post chicane } \\
\text { bunch length }\end{array}$ & $350 \mathrm{fs}$ \\
\hline $\begin{array}{c}\text { focused } \\
\text { beam size }\end{array}$ & $\begin{array}{c}x \approx 20 \mu \mathrm{m} \\
y \approx 50 \mu \mathrm{m}\end{array}$ \\
\hline energy spread & $0.2 \%$ \\
\hline $\begin{array}{c}\text { normalized } \\
\text { emittance }\end{array}$ & $5 \mathrm{~mm} \mathrm{mrad}$ \\
\hline
\end{tabular}
will deliver $\sim 310^{14}$ photons per pulse, with a pulse energy of $13.3 \mu J$. The priorities for this machine are to gain experience of operating a photo-injector gun and superconducting linac; to produce and maintain high- 
brightness electron beams; to achieve energy recovery from an FEL-disrupted beam and to study important synchronization issues, all of which are contributing towards the design of a next generation light source.

\subsection{MULTI 10 TW-LASER}

The customized tabletop chirped pulse amplification (CPA) multi $10 \mathrm{TW}$-laser system (COHERENT) - installed at the high field laser facility at Daresbury contains an ultra-short, band-width limited Kerr-lens mode locked (Spence et al., 1991; Brabec et al. 1992; Stingl et al.; 1995) Ti:Sapphire master oscillator (Micra; $\Delta \lambda>100 \mathrm{~nm}$ ) with a repetition rate of $81.25 \mathrm{MHz}$, followed by a stretcher and a regenerative amplifier $(2.8 \mathrm{~mJ} @ 1 \mathrm{KHz})$ which is used as a front-end system (Fig. 2) for a 4-pass Ti:Sa power amplifier. The output of the master oscillator exhibits a broad spectrum centered at $800 \mathrm{~nm}$. The pulses are then passed through a one to one imaging single grating stretcher to obtain a pulse-width of about $150 \mathrm{ps}$ before they are injected as seed in the regenerative amplifier (Legend) which is pumped by a diode-pumped, intra-cavity doubled, Q-switched Nd:YLF laser (Evolution). The customized power amplifier which contains a large aperture Ti:Sa crystal (Fig. 3, left), pumped from both ends (Relay imaged) using two spatially optimized frequency doubled Nd:YAG lasers (Powerlite Plus) operating at $10 \mathrm{~Hz}$, amplifies the pulses up to $1.5 \mathrm{~J}$ in a bow-tie configuration before recompression (Fig. 3, right).

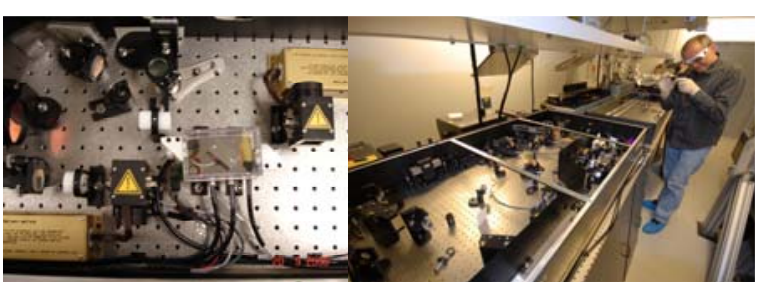

Fig. 2. Installation of the regenerative amplifier front end including pulse-picker and $\mathrm{kHz}$ compressor.

For future applications as laser driven plasma sources and particle acceleration, a pulse cleaner (Divall \& Ross, 2004; Priebe, 2006) using a fast pockels-cell driven by a KENTECH fast pulse generator will be established. The Legend incorporates an additional compressor to use the recompressed pulses $(2.5 \mathrm{~mJ} @ \mathrm{kHz})$ for further applications. One of the applications of the $\mathrm{kHz}$ beam will be in the development of non-destructive longitudinal, i.e. temporal diagnostics of the electron bunch; the bunch Coulomb field can interact with a probe laser pulse in an intra beam-line non-linear crystal, through the electro-optic effect, and temporal evolution of the Coulomb field is encoded into the probe as a time dependent probe polarization (Berden et al., 2004; Jamison et al., 2006). The $\mathrm{kHz}$ beam also drives a standalone $\mathrm{THz}$ source, with the $\mathrm{THz}$ produced through either optical rectification or a large area photo-conductive antenna; this $\mathrm{THz}$ source provides radiation pulses that can mimic the relativistic Coulomb field, and hence allow significantly simplified testing of diagnostic concepts (Jamison et al., 2003).

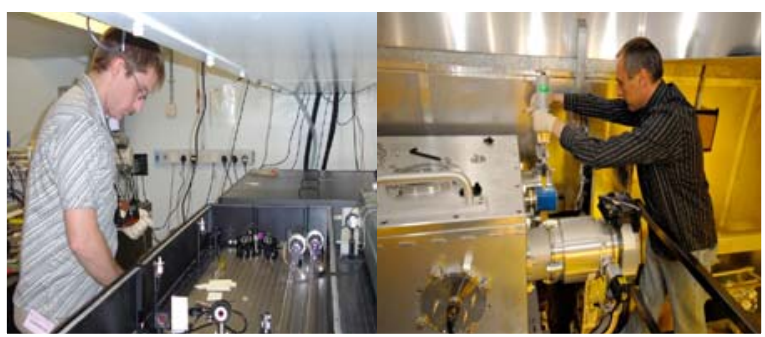

Fig. 3. Installation of the multi-pass power amplifier (left) and the chirped pulse compressor (right).

The detailed laser parameters - measured during the installation at Daresbury - of the multi $10 \mathrm{TW}$-laser system are given in Table 2 .

Tab. 2. The main laser parameters of the customized (COHERENT) tabletop multi $10 \mathrm{TW}$ Ti:Sa laser system installed at Daresbury high field laser laboratory.

\begin{tabular}{|c|c|c|}
\cline { 2 - 3 } \multicolumn{1}{c|}{} & $\mathrm{kHz}-$ Laser & TW-Laser \\
\hline wavelength & $798 \mathrm{~nm}$ & $802 \mathrm{~nm}$ \\
\hline bandwidth & $30 \mathrm{~nm}$ & $13 \mathrm{~nm}$ \\
\hline $\begin{array}{c}\text { energy before } \\
\text { compression }\end{array}$ & $2.8 \mathrm{~mJ}$ & $1500 \mathrm{~mJ}$ \\
\hline $\begin{array}{c}\text { energy after } \\
\text { compression }\end{array}$ & $2.2 \mathrm{~mJ}$ & $860 \mathrm{~mJ}$ \\
\hline pulse width & $35 \mathrm{fs}$ & $98 \mathrm{fs} / 35 \mathrm{fs}$ \\
\hline strahl ratio & & $0.6-0.8$ \\
\hline $\mathrm{M}^{2}$ & $01.2 x ; 1.11 \mathrm{y}$ & $1.38 x ; 1.45 \mathrm{y}$ \\
\hline
\end{tabular}

\section{INVERSE COMPTON SCATTERING}

The discovery of the Compton effect (Compton, 1923) was a milestone in physics in which the quantum nature of light was finally proved. The Compton effect is the inelastic scattering of X-rays from electrons. The electrons gain energy in the process and the Compton scattered X-rays are observed at lower energy than the incident X-rays. Due to the kinematics of the scattering process, there is a fixed relationship between the energy of the scattered X-rays and the angle through which they are scattered. Soon after discovery of the Compton effect, it was realized that electron motion within the scatterer Doppler broadens the emission spectrum - at fixed scattering angle - and it is this Doppler shift that forms the basis for inverse Compton X-ray sources. In 
inverse Compton scattering, the electrons are highly energetic and the Doppler shift results in the scattered photon gaining significant energy from electrons. This allows low energy photons (from an intense laser) when scattered by ultra relativistic electrons in an accelerator to generate hard X-rays. If the energy of the incident photon $E_{p h}$ in the frame of the interaction is much less that $m_{e} c^{2}$, the Thomson scattering cross section $\left(\sigma_{T h}=(8 \pi / 3) r_{e}^{2}\right)$ can be used to describe the probability of scattering, where $m_{e}$ is the rest mass of the electron and $r_{e}$ is the classical electron radius. In the laboratory frame the scattered laser photons are relativistically up shifted in frequency into the hard $\mathrm{X}$-ray range, and are emitted into a narrow cone. Alternatively the interaction can be viewed in the electron rest mass frame, the incident laser pulse appears as an electromagnetic undulator of wavelength $\lambda_{u}=\lambda_{p h} / \gamma(1$ $\beta \cos \Phi)$, where $\gamma$ is the relativistic factor $E_{e} / m_{e} c^{2}, \beta$ is the velocity of the electrons relative to the speed of light and $\Phi$ is the angle of incidence between electron and laser beam. The undulating electrons radiate photons, which are up shifted back into the laboratory frame by a second factor of $2 \gamma$. In the laboratory frame the X-rays are confined to a narrow cone with opening angle about $1 / \gamma$ in the electron beam direction. The Xray energy $E_{\gamma}$ varies with the observation angle $\theta$ in the laboratory frame due to the kinematics of the scattering

$$
E_{\gamma}=\frac{2 \gamma^{2}(1-\beta \cos \Phi)}{1+\frac{a_{0}{ }^{2}}{2}+\gamma^{2} \theta^{2}} E_{p h},
$$

where $a_{o}=e E_{p h} \lambda_{p h} / 2 \pi m_{e} c^{2}$ is the normalized vector potential of the laser field, analogous to the undulator deflection parameter $K=e B_{0} \lambda_{u} / 2 \pi m_{e} c^{2}$ of a static field undulator (Brown \& Kibble, 1964; Sarachik \& Schappert, 1970; Alferov et al., 1974; Coisson, 1979; Kim, 1989). The peak X-ray energy for transverse interaction $(\Phi=\pi / 2)$ is half for head on collisions $(\Phi=\pi)$. Due to the similarity between synchrotron radiation and inverse Compton scattering the radiation is often called laser synchrotron radiation or Compton synchrotron radiation. Using this analogy, and replacing the magnetic undulator with a laser beam of period $10^{4}$ times smaller, the electrons are deflected many more times and contribute to coherent energy gain with electron beams 100 times less energetic than in a conventional magnetic undulator. When $a_{0} \ll 1$, the interaction is in the linear regime; when $a_{0} \geq 1$ the interaction is in the nonlinear regime with higher order harmonics which may be used to extend the tuning range of the X-ray source (Ride et al., 1995; Salamin \& Faisal, 1996). However, ponderomotive scattering (Krafft, 2004; Krafft, 2005) also starts to occur for $a_{0}>1$, where the electrons are deflected from the laser focus point before they are able to scatter the photons therefore decreasing the total flux of emitted X-rays (Chen et al., 2008). The precise intensity and spectral profile of the resultant X-ray beam is closely linked to the laser frequency and bandwidth, the electron beam emittance, energy, and energy spread, and the interaction and focusing geometries (Hartemann et al., 2001). A schematic illustration of the inverse Compton scattering geometry is shown in Figure 4.



Fig. 4. Interaction geometry of inverse Compton scattering.

In the particle point of view the photon strikes an electron of the energy $E_{e^{-}}$with an angle of incidence $\Phi$. In the electron rest frame the energy of the photon is Doppler up-shifted to $E_{p h}=\gamma(1+\cos \Phi) E_{p h}$ and the wavelength of the scattered photon is given by the Compton formula $\lambda_{y^{\prime}}-\lambda_{p h^{\prime}}=\lambda_{c}\left(1-\cos \theta^{\circ}\right)$, where $\lambda_{c}=h / m_{e} c$ is the Compton wavelength, $h$ Planck's constant and $\theta^{\prime}$ is the scattering angle in the electron rest frame. The scattered photons gain energy $E_{\gamma^{\prime}}=m_{e} c^{2} E_{p h^{\prime}} /\left[m_{e} c^{2}+(1-\cos \theta) E_{p h}\right]$, with the contracted angle $\cos \theta^{\circ}=(\cos \theta-\beta) /(1-\beta \cos \theta)$ satisfying the momentum conservation constraint. Since the relativistic Doppler up-shifted X-ray energy $E_{p h}$ is much less that $m_{e} c^{2}$ in the electron rest frame, the centre of momentum frame is very close to that of the relativistic electron.

Using the back transformation in the laboratory frame $E_{\gamma}=\gamma(1-\beta \cos \theta) E_{\gamma}$, results in the relation between the scattered photon energy $E_{\gamma}$ to that of the incident laser photon energy $E_{p h}$ and electron energy $E_{e^{-}}$: $\mathrm{E}_{\gamma}=\mathrm{E}_{\mathrm{ph}}(1-\beta \cos \Phi) /\left[(1-\beta \cos \theta)+\mathrm{E}_{\mathrm{ph}} \quad(1-\beta \cos \theta-\right.$ $\left.\Phi) / \mathrm{E}_{\mathrm{e}^{-}}\right]$. The dependence of the energy of the scattered $\mathrm{X}$-ray photons on the collison angle $\Phi$ between the laser photons and the electrons, and on the scattering angle $\theta$, is shown in Figure 5, for the laser and electron parameters (Tab. 1 and Tab. 2). 


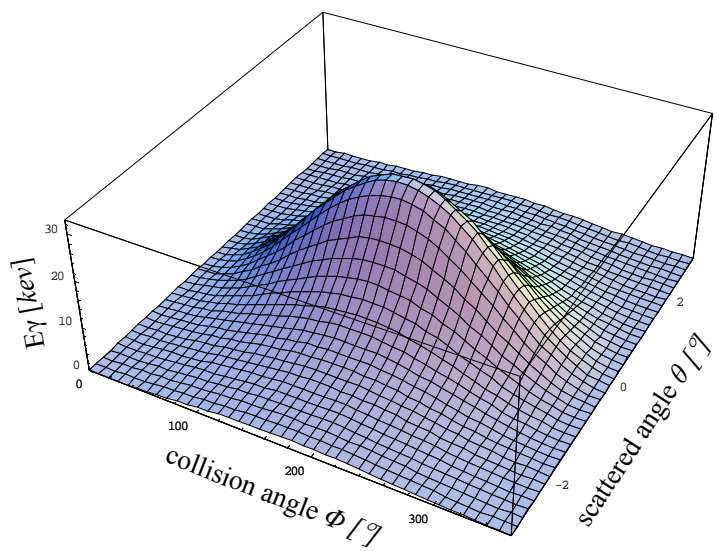

Fig. 5. Dependence of the X-ray energy $E_{\gamma}$ on the interaction geometry - the collision angle $\Phi$ - and the scattering angle $\theta$.

For a fixed laser incidence angle, there is a unique relation between the resultant scattered energy $E_{\gamma}$ and the photon trajectory of the X-rays. The peak X-ray energy $(\theta=0)$ for head on collisions $(\Phi=\pi)$ and transverse interaction $(\Phi=\pi / 2)$ simplifies - using $E_{p h} \ll m_{e} c^{2}, \beta \approx 1$ and $\gamma=\left(1-\beta^{2}\right)^{-1 / 2}-$ to the expected result:

head on collision: $\left.\quad E_{\gamma}\right|_{\Phi=\pi} ^{\theta=0} \approx 4 \gamma^{2} E_{p h} \approx 30 \mathrm{keV}$

transverse collision: $\left.E_{\gamma}\right|_{\Phi=\pi / 2} ^{\theta=0} \approx 2 \gamma^{2} E_{p h} \approx 15 \mathrm{keV}$.

In practice, the X-ray spectrum is broadened by the bandwidth and the divergence of the laser pulse as well

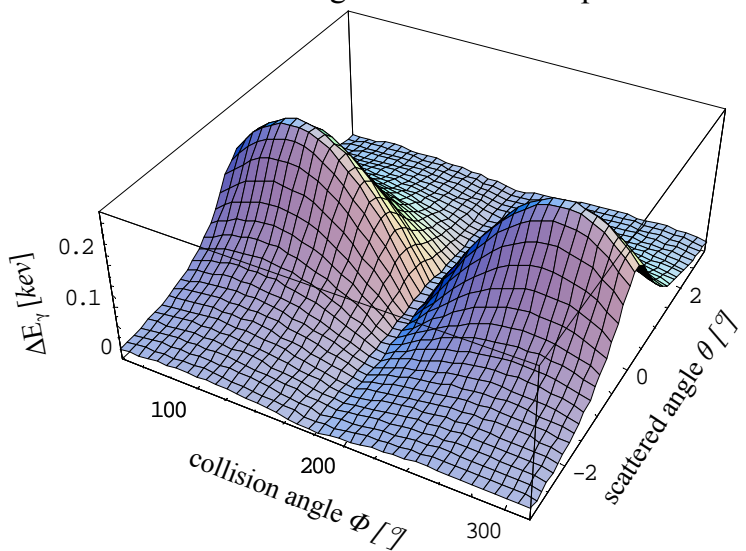

as the energy spread and the divergence of the electron bunch (Tab. 3).

Tab. 3. X-ray energy broadening induced by the wavelength bandwidth and the divergence of the laser pulse as well as the energy spread, and the divergence of the electron bunch.

\begin{tabular}{|c|c|c|}
\cline { 3 - 3 } \multicolumn{2}{c|}{} & $\Delta E_{\gamma} / E_{\gamma}[\%]$ \\
\hline laser & wavelength bandwidth & 1.2 \\
\cline { 2 - 3 } pulse: & divergence $[\Phi=\pi \mid \Phi=\pi / 2]$ & $0.004 \mid 1.8$ \\
\hline electron & energy spread & 0.4 \\
\cline { 2 - 3 } bunch: & divergence $[\Phi=\pi \mid \Phi=\pi / 2]$ & $0.0003 \mid 0.4$ \\
\hline
\end{tabular}

The energy spread depends linearly on the laser energy spread and quadratically on the electron energy spread. The energy spread of the bandwidth limited laser pulse is given by $\Delta E_{p h}=c h \Delta \lambda / \lambda^{2}$, where $\Delta \lambda$ is given by the time band-width product $\tau_{p h} c \Delta \lambda / \lambda^{2}=2 \ln 2 / \pi$ if the pulse shape is Gaussian and where $\tau_{p h}$ is the pulse duration of the laser pulse. For a $100 \mathrm{fs}$ laser pulse the energy spread is therefore $\Delta E_{p h} / E_{p h} \approx 1.2 \%$ and for a $30 f_{S}$ laser pulses $\Delta E_{p h} / E_{p h} \approx 3.9 \%$. Using an off axis parabolic mirror with a focal length $f$ of $1780 \mathrm{~mm}$ for head on interaction and $1230 \mathrm{~mm}$ for transverse interaction to focus the laser beam gives a focused spot size $(2 \lambda f / \pi \varnothing)$ of $\approx 40 \mu \mathrm{m}$ with an opening angle of $\approx 1.4^{\circ}$ and a focal length (twice the Raleigh length $\left.z_{R}=\pi w o 2 / \lambda\right)$ of $\approx 3 \mathrm{~mm}$ for head on collision and a spot size of $\approx 28 \mu \mathrm{m}$ with an opening angle of $\approx 2.1^{\circ}$ and a focal length of $\approx 1.5 \mathrm{~mm}$ for transverse collision.

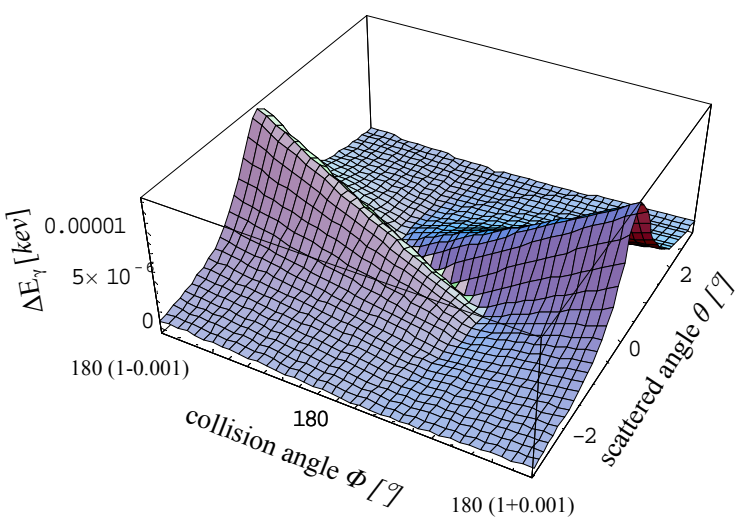

Fig. 7: X-ray energy spread $\Delta E_{\gamma}$ caused due to the divergence of the laser beam as a function of the collision angle $\Phi$ and the scattering angle $\theta$ (left) and the detailed distribution of the X-ray energy spread $\Delta E_{\gamma}$ for head on collision geometry (right).

The divergence of the electron bunch is $\approx 0.2^{\circ}$ is given by the normalized emittance and the spot size $\sigma_{e}$ of the electron focus $\left(\Delta \theta_{e}=\varepsilon_{n} / \gamma \beta \sigma_{e}\right)$. In head on collision the on axis X-ray energy spread caused by the 
divergence of the photons and of the electrons is negligible $(0.004 \% \mid 0.0003 \%)$ compared with the broadening due to their own energy spread (Fig. 6). For transverse interaction the divergence of the photons becomes the dominating factor $(1.8 \%)$ and the energy spread induced from the divergence of the electron $(0.4 \%)$ becomes comparable to the energy spread of them selves.

In the rest frame of the electron bunch, the differential scattering cross section is described by the Klein-Nishina formula (Klein \& Y. Nishina, 1929):

$$
\frac{d \sigma_{c}}{\sin \theta^{\prime} d \theta^{\prime}}=\pi r_{e}^{2} \varepsilon^{\prime 2}\left[\varepsilon^{\prime}+\frac{1}{\varepsilon^{\prime}}-1+\left(\cos \theta^{\prime}\right)^{2}\right],
$$

where $\varepsilon$ is the ratio between the energies of the scattered and incident photon $\varepsilon^{\prime}=E_{\gamma} / E_{p h}$. If the interaction is such that the recoil momentum of the electron can be disregarded $E_{p h} \ll \mathrm{m}_{\mathrm{e}} \mathrm{c}^{2}\left(\varepsilon^{\prime}=1\right)$ leads to the well-know $(\cos \theta)^{2}$ distribution of dipole radiation, the differential Thompson scattering cross section:

$$
\frac{d \sigma_{T h}}{d \theta^{\prime}}=\pi r_{e}^{2}\left[1+(\cos \theta)^{2}\right] .
$$

Integrated, gives the Thompson cross section $\sigma_{T h}=(8 / 3) \pi r_{e}^{2}=0.66510^{-28} \mathrm{~m}^{2}$ for elastic scattering of a photon from an electron. The total cross-section for the non-relativistic $\varepsilon^{\prime} \gg 1$ and the extremely relativistic $\varepsilon^{`} \ll 1$ cases are given as:

$$
\begin{array}{ll}
\sigma \approx \sigma_{T h}\left(1-2 \varepsilon^{\prime}+\frac{26 \varepsilon^{\prime 2}}{5}+\cdots\right), & \text { for } \quad \varepsilon^{\prime}>>1, \\
\sigma \approx \frac{3}{8} \sigma_{T h} \frac{1}{\varepsilon^{\prime}}\left(\ln 2 \varepsilon^{\prime}+\frac{1}{2}\right), & \text { for } \quad \varepsilon^{\prime}<<1 .
\end{array}
$$

Transforming the differential Klein-Nishina cross section (5) to the laboratory frame of reference using of the above mentioned boundary condition as well as its derivation $\left(d \theta \theta^{`} \sin \theta^{`}=d \theta \sin \theta\left(1-\beta^{2}\right) /(1-\beta \cos \theta)^{2}\right)$ and the transformations $E_{\gamma^{\prime}} \rightarrow E_{\gamma}$ and $\mathrm{E}_{\mathrm{ph}}{ }^{\prime} \rightarrow \mathrm{E}_{\mathrm{ph}}$ gives the differential the Compton cross section:

$$
\frac{d \sigma_{c}}{\sin \theta d \theta}=\pi r_{e}^{2} \varepsilon^{2}\left[\varepsilon+\frac{1}{\varepsilon}-1+\left(\frac{\cos \theta-\beta}{1-\beta \cos \theta}\right)^{2}\right],
$$

with $\varepsilon=m_{e} c^{2} /\left[m_{e} c^{2}+\gamma(1+\cos \Phi)(1-(\cos \theta-\beta) /(1-\right.$ $\left.\beta \cos \theta)) E_{p h}\right]$. The cross section for scattering a photon into a cone of angle $\theta_{c}$ in the laboratory frame is then $\sigma_{c}\left(\theta_{c}\right)=\int d \theta\left(d \sigma_{c} / d \theta\right)$ and the average energy of
X-rays backscattered in the laboratory frame is $\left(\int d \theta E_{\gamma} d \sigma_{c} / d \theta\right) /\left(\int d \theta d \sigma_{c} / d \theta\right)$. Limiting the integration to the opening half angle of $1 / 2 \gamma$ leads to a scattering cross section independent of the machine energy. Numerically this is $0.165 \mathrm{barn}$, of course integrating over the full phase space gives the Thomson cross section of 0.665 barn. The total number of X-ray photons $N_{\gamma}$ scattered per unit time and volume into a cone of angle $\theta_{c}$ is given by the product of the electron beam 4-current $j_{\mu}\left(x_{\nu}\right)=e c n_{e}\left(x_{\nu}\right) u_{\mu} / \gamma$ and the incident photon 4-flux $\Phi_{\mu}\left(x_{\nu}\right)=c n_{\lambda}\left(x_{\nu}\right) k_{\mu} / \omega$ as (Landau \& Lifshitz, 1975):

$$
\begin{aligned}
d^{4} N_{\gamma}\left(x_{\nu}\right) / d^{4} x_{\nu} & =\left(\sigma_{c} / e c c\right) j_{\mu}\left(x_{\nu}\right) \Phi_{\mu}\left(x_{\nu}\right) \\
& =\left(c \sigma_{c} / \gamma \omega\right) n_{e}\left(x_{\nu}\right) n_{p h}\left(x_{\nu}\right) u_{\mu} k^{\mu} .
\end{aligned}
$$

Here, $u_{\mu}=d x_{\nu} / c d t$ is the electron 4-velocity, $k^{\mu}=(\omega / c, k)$ is the incident photon 4 -wave number. The number of X-ray photons per unit time can be expressed in Cartesian coordinates explicitly as $d^{4} N_{\gamma}(x, y, z, t) / d x d y d z c d t=\sigma_{c} n_{e}(x, y, z, t) n_{p h}(x, y, z, t)(1-\beta \cos \Phi)$ (Hartemann et al., 2005),

$$
N_{\gamma}=(1-\beta \cos \Phi) \sigma_{c} L
$$

where $L=\int n_{e} n_{p h} d x d y d z c d t$ is the luminosity which is determined by the collision geometry of the electron bunch and the laser pulses. In order to evaluate the number pulse length of X-rays we assume that both the electron bunch and the laser pulse satisfy the Gaussian distribution. The density profile of the laser light pulse and the electron bunch is thus given by:

$$
\begin{aligned}
& n_{p h}=\frac{N_{p h}}{2 \sqrt{2} c \pi^{3 / 2}} \frac{1}{w_{p h x}[z] w_{p h y}[z] \tau_{p h}} \\
& \exp \left[-\frac{2 x^{2}}{w_{p h x}^{2}[z]}-\frac{2 y^{2}}{w_{p h y}^{2}[z]}-\frac{2\left(t-\frac{z}{c}\right)^{2}}{\tau_{p h}^{2}}\right] \\
& n_{e}=\frac{N e}{2 \sqrt{2} c \pi^{3 / 2}} \frac{1}{w_{e x} w_{e y} w_{e z}} \\
& \exp \left[-\frac{x^{2}}{2 w_{e x}{ }^{2}}-\frac{y^{2}}{2 w_{e y}{ }^{2}}-\frac{\left(t+\frac{z}{\beta c}\right)^{2}}{2 w_{e z}{ }^{2}}\right] .
\end{aligned}
$$

$w_{p h x, y}=\left(w_{0}^{2}\left(1+\left(z / z_{r}\right)^{2}\right)\right)^{1 / 2}$ is the beam waist, $N_{p h}$ the total number of photons in the laser pulse, $\tau_{p h}$ the pulse duration and is related to the bandwidth as $\Delta \tau_{p h} \Delta \omega=\sqrt{2}$, in the case of a Fourier-transform-limited pulse, $w_{0}$ the $1 / e^{2}$ focal radius, $N_{e}$ the total number of electrons in one bunch, $w_{e x, y}$ are the magnitude to the 
electron bunch in the $x$ and $y$ directions and $c w_{e z}$ is the bunch length. For laser pulses crossing the electron bunch at an angle $\Phi$ the luminosity could be described as: $L=N_{e} N_{p h} /\left[2 \pi\left(w_{e y}{ }^{2}+w_{o y}{ }^{2} / 4\right)^{1 / 2}\left(\cos ^{2} \Phi / 2\left(w_{e x}{ }^{2}+w_{o x}{ }^{2} / 4\right)+\right.\right.$ $\left.\left.\left(w_{e z}{ }^{2}+c^{2} \tau_{p h}{ }^{2} / 4 \beta^{2}\right) \sin ^{2} \Phi / 2\right)^{1 / 2}\right]$ (Yang et al., 1999). This leads to a luminosity of $L=N_{e} N_{p h} /\left[2 \pi\left(w_{e x}^{2}+w_{o x}^{2} / 4\right)^{1 / 2}\right.$ $\left.\left(w_{e y}{ }^{2}+w_{o y}^{2} / 4 \beta^{2}\right)^{1 / 2}\right]$ for head on collision and respectively $L=N_{e} N_{p h} /\left[2 \pi\left(w_{e y}{ }^{2}+w_{o y}{ }^{2} / 4\right)^{1 / 2}\left(w_{e z}{ }^{2}+c^{2} \tau_{p h}{ }^{2} / 4 \beta^{2}\right)^{1 / 2}\right]$ for transverse collision. The total number of scattered X-ray photons $N_{\gamma}$ per unit time and volume into a cone of angle $\theta_{c}$ is given as:

$$
\begin{aligned}
& N_{\gamma} \approx 9 \cdot 10^{7} \frac{\mathrm{X} \text {-ray photons }}{\text { shot }} \text { for head on collision and } \\
& N_{\gamma} \approx 8 \cdot 10^{6} \frac{\mathrm{X} \text {-ray photons }}{\text { shot }} \text { for transverse collision, }
\end{aligned}
$$

where the electron bunch was taken as focused to an spot size of $w_{e x}=35 \mu \mathrm{m}, w_{e y}=20 \mu \mathrm{m}$ and $w_{e z}=c 350 \mathrm{fs}$ and the laser focused to a spot size of $w_{0}=40 \mu \mathrm{m}$ with a pulse duration of $100 \mathrm{fs}$.

In head on geometry the overlap integral depends only on the spot size, and the laser and electron bunch duration plays no role. The laser pulse travels through the electron bunch and the X-ray pulse length is determined almost entirely by the length of the electron bunch. In transverse interaction it is noticeable from the interaction geometry (Fig. 4) that the electron bunch spot size in $x$-direction $w_{e x}$ is negligible, but the beam size in $y$-direction $w_{e y}$ must fit with the laser spot size and the electron bunch length $w_{e z}$ must be comparable to the traveling time of the laser pulse through the electron bunch $c \tau_{p h}$. The length of the X-ray pulse will be dependent on how long the laser and electron beam interact. This indicates that tight beam focusing and short laser pulses will result in short X-rays due to a minimized interaction time.

The relation between these parameters and the resultant source pulse $\tau_{\gamma}$ length is expressed for head on collision as $w_{e z} / c \beta$ and as $w_{e z}\left(w_{e y}{ }^{2}+c^{2} \tau_{p h}{ }^{2} / 4 \beta^{2}+w_{o y}{ }^{2} / 4\right)^{1 / 2}$ $\left[c\left(w_{e y}{ }^{2}+w_{e z}{ }^{2}+w_{o y}{ }^{2}+w_{o y}{ }^{2} / 4+c^{2} \tau_{p h}{ }^{2} / 4 \beta^{2}\right)^{1 / 2}\right]$ respectively for transverse collision (Pogorelski et al., 2000).

The $\mathrm{T}^{3}$ laser beam transport line is shown in Figure 7. The vacuum system pumping, gauging and protection (fast valve) is designed to operate assuming no window.
This is necessary as a window thick enough to withstand atmospheric let-up would be radiation damaged by absorption of terawatt pulses, and a thin window will have the risk of breakage and glass particle contamination of ERLP during a vacuum failure.

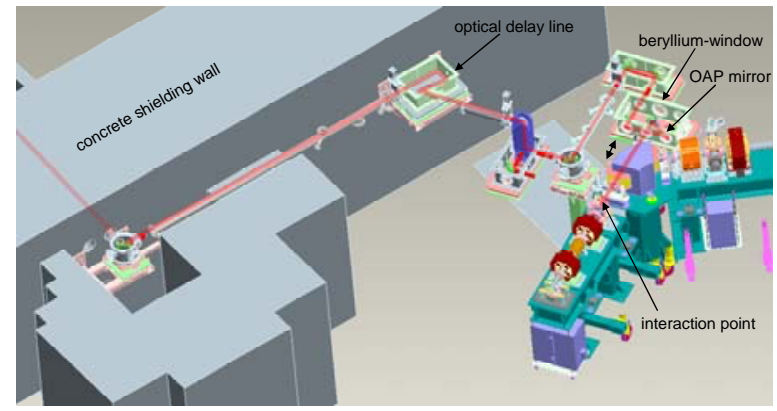

Fig. 7. Laser beam transport line through the concrete shielding wall to the interaction region.

The laser area vacuum design specification is at $10^{-6} \mathrm{mbar}$ and the ERLP vacuum is at $10^{-8} \mathrm{mbar}$. The ERLP system is protected from a let-up in the beam line by a fast acting valve. The laser beam propagates from the CPA compressor vessel through a concrete shielding wall passing an optical delay line, periscoped down to the electron beam level, focused via an off axis parabola mirror (OAP) and finally turned to the interaction point. For the case of a transverse interaction the periscope is also used to rotate the polarization of the laser. The last vacuum vessel containing the OAPmirror sits on rails allowing the focal position to be moved through the electron bunch. The focal length of the OAP-mirror is $1780 \mathrm{~mm}$ in head on collision and $1230 \mathrm{~mm}$ for transverse interaction. The scattered hard $\mathrm{X}$-rays - in the direction of the electron beam - will be taken through a beryllium-window to the diagnostic area (X-Ray deep depletion CCD camera, fast X-ray streak camera, fast melting experiment) out off the machine vacuum. In head on collision, the last turning mirror has a hole of about $5 \mathrm{~mm}$ diameter to let the $\mathrm{X}$-rays through and also to take a part of the laser beam out for pump probe applications. Figure 8 shows the designed experimental setup for the head on collision (left) and the transverse collision (right). 

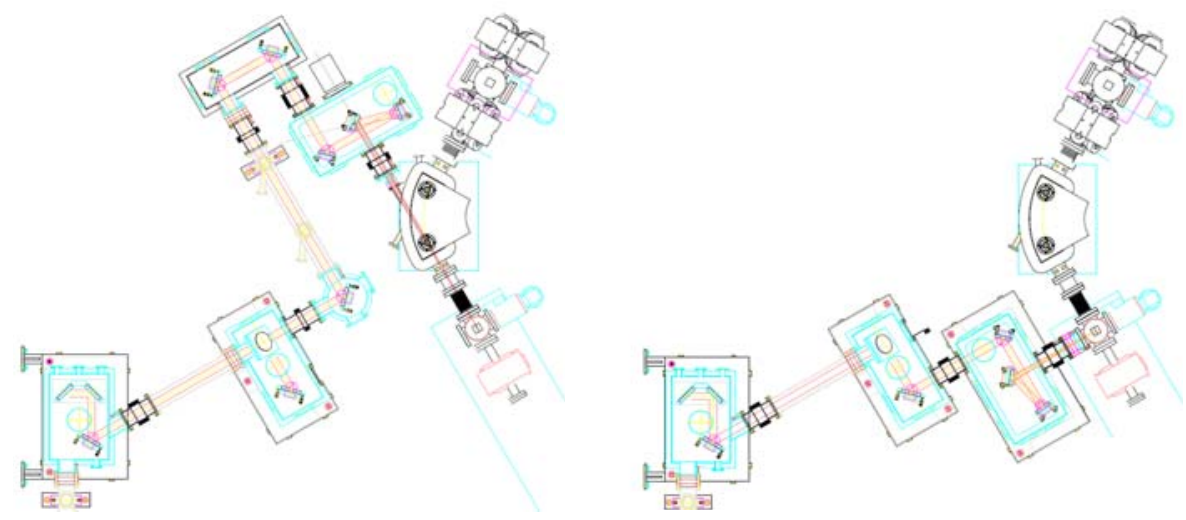

Fig. 8. Designed experimental setup for head on collision (left) and transverse collision (right).

In order to model the X-ray output, it was first necessary to simulate the electron bunch spatial, angular and energy distribution at the interaction point. For optimal generation of Compton scattered X-rays, the ERLP lattice is set to focus the electrons to give the highest electron density at the interaction point. This maximizes the X-ray flux and also reduces the size of the X-ray source. The electron trajectories were modeled using the particle tracking code ELEGANT. In excess of 100,000 electrons were tracked through to the focus. The electron distribution produced was then used as input to code written to simulate the inverse Compton scattering. The electron data consisted of the electron position at the focus plane, the electron direction vector (direction cosines) and the electron energy. The laser photons were taken as a



Fig. 9. X-ray energy $E_{\gamma}$ versus the emission angle $\theta$ in head on scattering geometry.
Gaussian angular distribution (in horizontal and vertical planes) and an energy distribution given by the transform function of the laser Gaussian temporal distribution. For each electron at the focus, a laser photon was generated at random by the code with direction vector and energy randomly chosen according to their assumed distributions. An X-ray was then generated with direction vector selected randomly conforming to the Klein-Nishina cross section. The calculated X-ray energy as a function of emission angle in head on scattering geometry is shown in Figure 9 and for side scattering geometry in Figure 10, respectively. The spectral brightness of the X-ray source (photons $/ \mathrm{mm}^{2} / \mathrm{mrad}^{2} / \mathrm{s} / 0.1 \%$ bandwidth) is shown in Figure 11. Figure 12 shows the angular distribution of the X-rays for head on collision.



Fig. 10. X-ray energy $E_{\gamma}$ in dependence on the emission angle $\theta$ in side scattering geometry. 


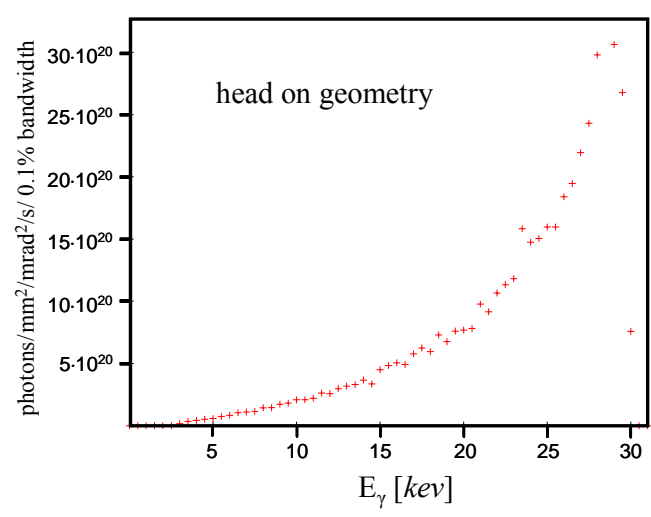

Fig. 11. Spectral brightness of the X-ray source versus $E_{\gamma}$ for head on scattering.

\section{CONCLUSION AND OUTLOOK}

COBALD is an instrument capable of generating a high peak brightness $f_{S} \mathrm{X}$-ray pulses. X-rays generated by the interaction of the table top multi 10 -TW laser with the electron bunch have been modeled by Monte Carlo simulation calculating over $10^{5}$ interactions, and have shown that brightness in excess of $10^{21} \mathrm{X}$ rays $/ \mathrm{mm}^{2} / \mathrm{mrad}^{2} / \mathrm{s} / 0.1 \%$ bandwidth will be obtained in back scattering geometry. It is intended to operate COBALD in both counter-propagating and transverse interaction geometry. The peak X-ray energy is about $30 \mathrm{keV}$ in backscattering and approximately $15 \mathrm{keV}$ in side scattering geometry. The X-ray pulse length in back scattering geometry will be about $350 f_{s}$ and in side scattering geometry about $100 \mathrm{fs}$. The X-ray source size will be of order $20 \times 35 \mu^{2}$. Characterization of the $\mathrm{X}$-ray beam such as its profile and energy spectrum will provide vital information about the spatial and temporal structure of the electron beam in the ERLP. Further studies are planned to examine photon/electron beam synchronization and source stability; this is a necessary requirement prior to any dynamic experiments are performed. The availability of this ultra short brilliant X-ray source will allow X-ray diffraction and spectroscopy techniques to be extended into the time domain allowing atomic resolution probing of structural dynamics. This type of experiment could be carried out using a pump pulse - for example by extracting a fraction of the multi 10-TW laser pulse - to start a time dependent process in a sample. The sample could then be probed, after a short delay, by the X-ray pulse. This would allow, for example, intermediate species to be probed during chemical reactions or strain relaxation following lattice distortion to be measured.

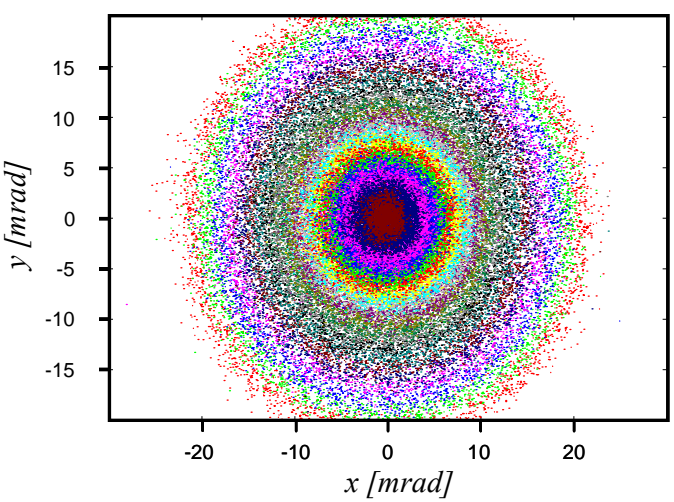

Fig. 12. Angular distribution of the $\mathrm{X}$-rays generated in head on collision; each color is a $1 \mathrm{keV}$ energy band with $20-21 \mathrm{keV}$ on outside and $30-31 \mathrm{keV}$ at centre.

\section{ACKNOWLEDGEMENT}

We would like acknowledge the financial support of the Northwest Development Agency - through the Northwest Science Fund -,the Central Laser Facility at Rutherford and the Science and Technology Facilities Council.

\section{REFERENCES}

Alferov, D.F., Bashmakov, Yu. AND Bessonov, E.G. (1974). Undulator Radiation. Sov. Phys. Tech. Phys. 18, 1337.

Brown, L.S. AND KiBBLE, T.W.B. (1964). Interaction of Intense Laser Beams with Electrons. Phys. Rev. 133, A705.

Brabec, T., SpielmanN, CH., Curley, P.F. AND Krausz, F. (1992). Kerr lens mode locking. Optics Letters 17 (18), 1292.

Bazerov, I., Belomestnykh, S., Bilderback, D., FinKelsteIn, K., FONTES, E., GRAY, S., GRUNER, S.M., KraffT, G.A., Merminga, H., HelmKe, R., Rogers, J., Sinclair, C., TAlman, R. AND Tigner, M. (2001). Study for a Proposed Phase I Energy Recovery Linac (ERL) Synchrotron Light Source at Cornell University; CHESS Technical Memo 01-003, JLAB-ACT-01-04.

Berden, G., JAMISON, S.P., MACLEOD, A.M., GILlESPIE, W.A., REDLICH, B. AND VAN DER MeER, A.F.G. (2004). Electro-Optic Technique with Improved Time Resolution for Real-Time, Nondestructive, Single-Shot Measurements of 
Femtosecond Electron Bunch Profiles, Phys. Rev. Lett. 93, 114802.

BORLAND, M. (2005). Evaluation of the possibility of upgrading the advanced photon source to an Energy Recovery Linac. Nucl. Instr. and Meth. A, for the ERL05 Workshop (2005).

Baker, S., Robinson, J.S., Haworth, C.A., Teng, H., SMITH, R.A., ChIRIL, C.C., LEIN, M., TISCH, J.W., MARANGOS, J.P. (2006). Probing proton dynamics in molecules on an attosecond timescale. Science 312 (5772), 4247.

CoIsson, R., (1979). Angular-spectral distribution and polarization of synchrotron radiation from a "short" magnet. Phys. Rev. A 20, 524.

Compton, A.H., (1923). A quantum theory of the scattering of X-rays by light elements. Phys. Rev., 21,207 , and 483.

Catravas, P., Esarey, E. AND LeEmans, W.P. (2001). Femtosecond Thomson scattering $\mathrm{X}$-ray source based on laser wakefield accelerator for ultrafast X-ray absorption spectroscopy. Meas. Sci. Technol. 12, 1828.

Chouffani, K., Harmon, F., Wells, D., Jones, J. AND LANCASTER, G. (2006). Laser-Compton scattering as a tool for electron beam diagnostics. Laser and Particle Beams, 24 (3), 411.

Chen, L.M., Kando, M., Xu, M.H., LI, Y.T., KogA, J., Chen, M., Xu, H., Yuan, X.H., DONG, Q.L., ShenG, Z.M., Bulanov, S.V., Kato, Y., ZhanG, J. AND TAJIMA, T. (2008). Study of X-Ray Emission: Enhancement via a High-Contrast Femtosecond Laser Interacting with a Solid Foil. Phys. Rev. Lett., 100, 045004.

Divall, E.J. AND Ross, I.N. (2004). High dynamic range contrast measurements by use of an optical parametric amplifier correlator. Optics Letters 29 (19), 2273.

Guo, T., SpielmanN, C., Walker, B.C. AND Barty, C.P.J. (2001). Roughness enhanced K x-ray emission from femtosecond laser produced copper plasmas. Rev. Sci. Instrum. 72, 41.

Gruner, S.M. AND Tigner, M. (Eds.), (2001). Study for a Proposed Phase I Energy Recovery Linac (ERL) Synchrotron Light Source at Cornell University; CHESS Technical Memo 01-003, JLAB-ACT-01-04.

Giulietti, D., Galimberti, M., Giulietti, A., Gizzi, L.A., LaBATE, L. AND TOMAssini, P. (2005). The laser-matter interaction meets the high energy physics: Laser-plasma accelerators and bright gamma X-ray sources. Laser and Particle Beams, 23 (3), 309.

Helliwell, J.R. AND RentzePis, P.M. (1997). TimeResolved X-ray and Electron Diffraction. Chap. 7 Time-resolved diffraction, Oxford Univ. Press.

Hartemann, F.V., Baldis, H.A., Kerman, A.K., Le Foll, A., LuHMANN, N.C., AND RuPP, B. (2001) Three-dimensional theory of emittance in Compton scattering and x-ray protein crystallography. Phys. Rev. E 64, 016501.

Hafz, N., LeE, H.J., KIM, G.H. AND SuK, H. (2003). Ultrashort X-ray source based on the nonlinear Thomson scattering of femtosecond lasers from plasma-accelerated electron beams. IEEE Trans. Plasma Science 31 (6), 1388.

Hartemann, F.V., Tremaine, A.M., Anderson, S.G., BARTY, C.P.J., BETTS, S.M., BOOTH, R., BROWN, W.J., Crane, J.K., Cross, R.R., Gibson, D.J., FitTinghoff, D.N., KubA, J., Le SAge, G.P., Slaughter, D.R., Wootton, A.J., Hartouni, E.P., SPRINGer, P.T., RosenzWeig, J.B. AND KERMAN, A.K. (2004). Characterization of a bright tunable ultrafast Compton scattering X-ray source. Laser and Particle Beams, Volume 22, Issue 03, Jul 2004, pp 221-244

HARTEMANN, F.V., BRown, W.J., GiBSON, D.J., ANDERSon, S.G., Tremaine, A.M., SPRINGER, P.T., WoOtTON, A.J., HARTOUNI, E.P. AND BARTY C.P.J. (2005). High-energy scaling of Compton scattering light sources; Phys. Rev. SPECIAL TOPICS - ACCELERATORS AND BEAMS 8, 100702.

YAnG, J., Washio, M., Endo, A., Hori; T. (1999). Evaluation of fs X-rays produced by Thomson scattering under linear and nonlinear interactions between a low-emittance electron beam and an intense polarized laser light. Nuclear Instruments and Methods in Physics Research A 428, 556.

Jamison, S.P., Shen, J., MacLeod, A.M., Gillespie, W.A., JAROSZYNSKI, D.A. (2003). High-temporalresolution, single-shot characterization of terahertz pulses. Optics Letters 28, 1710.

Janulweicz, K.A., Lucianetti, A., Priebe, G., NiCKLES, P.V. (2004). Review of state-of-the-art and output characteristics of table-top soft x-ray lasers. X-RAY SPECTROMETRY, 33 (4), 262266.

JANulewiCZ, K.A., TÜMMLER, J., PRIEBE G. AND NiCKLES P.V. (2005). Plasmakinetics perspective 
of a collisional Ni-like X-ray laser pumped by a single profilled laser pulse. Phys. Rev. A 72, 1.

JAMison, S.P., MACLEOD, A.M., BERDEN, G., JAROSZYNSKI, D.A., GILLESPIE, W.A. (2006). Temporally resolved electro-optic effect. Optics Letters 31, 1753.

KLEIN, O. AND NishinA, Y. (1929). Über die Streuung von Strahlung durch freie Elektronen nach der neuen relativistischen Quantendynamik von Dirac; Zeit. f. Phys., 52, 853.

KIM, K.J. (1989). in Physics of Particle Accelerators, edited by M. Month, Dienes, M., AIP Conf. Proc. 184, 565.

Kim, K.J., Chattopadhyay, S. And Shank, C.V. (1994). Generation of Femtosecond X-rays by 90 degrees Thomson Scattering. Nucl. Instr. And Methods for Physics Research, A 341, 351.

KuliPANOV, G.N., Skrinsky, A.N., VinOKUROV, N.A. (1998). J. Synchrotron Radiat. 5, 176.

KRAFFT, G.A. (2004). Saturated Spectral Distributions of Thomson-Scattered Photons from HighIntensity Pulsed Lasers. Phys. Rev. Lett, 92.

Krafft, G.A., Doyuran, A. AND Rosenzweig, J.B. (2005). Pulsed-laser nonlinear Thomson scattering for general scattering geometries. Phys. Rev. E 72, 056502 .

Khattak, F., Saiz, E.G., Dzelzainis, T., Riley, D. AND ZHAI, Z. (2007). Scale-length optimizing of short pulse $\mathrm{Cu} \mathrm{K}$-alpha laser plasma sources; Appl. Phys. Lett. 90.

LANDAU, L.D. AND LifSHITZ, E.M. (1975). Classical Theory of Fields. Butterworth-Heinemann, Oxford, UK, 4th ed..

Leemans, W., Volfbeyn, P., Zolotorev, M., Kim, K.J., Chattopadhyay, S., Schoenlein, R., BALling, P., SHANK, C.V., CHIN, A. AND Glover, E. (1996). Laser-based Sub-picosecond Electron Bunch Characterization Using 90 degrees Thomson Scattering. Phys. Rev. lett., 77, 4182.

Leemans, W.P., Esarey, E., Zholents, A., Chin, A., Schoelein, R., Shank, C., Chattopadhyay, S. (2000). Femtosecond X-ray Generation through Relativistic Electron-Beam-Laser Interaction. Comptes redus a l'Académie des Sciences, Serie IV, 279-296.

Li, Y., Huang, Z., Borland, M.D. And Milton, S. (2002). Small-angle Thomson scattering of ultrafast laser pulses for bright, sub-100-fs x-ray radiation. Physical Review STAB, vol. 5, 044701.
Lucianetti, A., Janulewicz, K.A., Kroemer, R., Priebe, G., Tummler, J., SANDNer, W., Nickles, P.V., REDKORECHEV, V.I. (2004). Transverse spatial coherence of a transient nickellike silver soft-x-ray laser pumped by a single picosecond laser pulse. Optics Letters, 29 (8), 881.

Legall, H., StIEl, H., ARKADIEV, V. AND BJEOUMIKHOV, A.A. (2006). High spectral resolution $\mathrm{X}$-ray optics with highly oriented pyrolytic graphite. Optics Express 14, 10.

Materlik, A.G., TAtChYN, R. AND Winick, H. (1995). The LCLS: A fourth generation light source using the SLAC linac; Rev. Sci. Instr. 66, 1987.

Neil, G.R., Behre, C., Benson, S.V., Bevins, M., Biallas, G., Boyce, J., Coleman, J., DillonTownes, L.A., Douglas, D., Dylla, H.F., Evans, R., Grippo, A., Gruber, D., Gubeli, J., HARDY, D., HERNANDEZ-GARCIA, C., JORDAN, K., Kelley, M.J., Merminga, L., Mammosser, J., Moore, W., Nishimori, N., Pozdeyev, E., Preble, J., Rimmer, R., Shinn, M., Siggins, T., TENNANT, C., WALKER, R., Williams, G.P. AND ZhANG, S. (2005). The JLab High Power ERL Light Source, The 32nd Advanced ICFA Beam Dynamics Workshop on Energy Recovering Linacs, Jefferson Lab, Newport News, Virginia.

Notley, M.M., Weber, R.L., Fell, B., JefFries, J., FREEMAN, R.R., MACKINNON, A.J., DICKSON, R., Hey, D., Khattak, F., Saiz, E.G.And Gregori, G. (2006). Development of time resolved x-ray spectroscopy in high intensity laser-plasma interactions Rev. of Sci. Instr. 77.

Pogorelski, I.V., ET AL. (2000). Physical Review STAB, vol. 3, 090702.

Phuoc, K.Ta., Rousse, A., Pittman, M., Rousseau, J.P., MalKa, V., Fritzler, S., Umstadter, D., AND Hulin, D. (2003). X-Ray Radiation from Nonlinear Thomson Scattering of an Intense Femtosecond Laser on Relativistic Electrons in a Helium Plasma. Phys. Rev. Lett. 91, 195001.

Poole, W., ET AL. (2003). Proc. of the 2003 Particle Accelerator Conf., Portland, or IEEE, Piscataway, NJ, 189.

PRIEBE, G., REDKORECHEV, V.I., JANULEWICZ K.A. AND NiCKLES P.V. (2006). Pulse shape measurement by a non-collinear third-order correlation technique. Optics Communications 259 (2), 848.

Ride, S.K., ESAREY, E. AND BAINE, M. (1995). Thomson scattering of intense lasers from electron 
beams at arbitrary interaction angles. Phys. Rev. E 52,5425 .

Ross, M.C. ET. AL. (1997). Proc. Beam Inst. Wkshp. 7, ed. A. Lumpkin, C.E. Eyberger.; New York:AIP Press, 281.

Riley, D., Angulo-Gareta, J.J., Khattak, F.Y. AND LAMB, M.J., Foster, P.S., DiVALl, E.J., HOOKER, C.J., LANGLEY, A.J., Clarke, R.J. AND NEEly, D. (2005). $\mathrm{K}_{\alpha}$ yields from Ti foils irradiated with ultrashort laser pulses. Phys. Rev. E, 71.

SARACHIK, E.S. AND SCHAPPERT, G.T. (1970). Classical Theory of the Scattering of Intense Laser Radiation by Free Electrons. Phys. Rev. D 1 (10), 2738.

Strickland, D. AND Mourou G. (1985). Compression of amplified chirped optical pulses. Optics Com. $56,219$.

Spence, D.E., KeAn, P.N. AND SibBett, W. (1991). 60fsec pulse generation from a self-mode-locked Ti:sapphire laser; Opt. Lett. 16, 42.

Stingl, A., LenzNer, M., Spielmann, Ch., Krausz, F. AND SZIPOCS, R. (1995). Sub-10-fs mirrordispersion-controlled Ti:sapphire laser. Optics Letters.. 20, 602.

Schoenlein, R.W., LeEMANS, W.P., Chin, A.H., Volfbeyn, P., Glover, T.E., Balling, P., ZolotoreV, M., KiM, K.J., ChatTopadhyay, S. AND ShanK, C.V. (1996). Femtosecond X-ray Pulses at $0.4 \AA$ Generated by $90^{\circ}$ Thomson Scattering: A Tool for Probing the Structural Dynamics of Materials, Science 274, 236.

SAlAmin, Y.I. AND FAISAL, F.H.M. (1996). Harmonic generation by superintense light scattering from relativistic electrons; Phys. Rev. A 54, 4383.

Siders, W., Cavalleri, A., Sokolowski-Tinten, K., Tóth, Cs., GuO, T., KAMMLER, M., Wilson, K.R., VON DER LINDE, D. AND BARTY, C.P.J. (1999). Detection of Nonthermal Melting by Ultrafast Xray Diffraction. Science 286 (5443), 1340.

Schoenlein, R., Chattopadhyay, S., Chong, H.H.W., Glover, E., Heimann, P., Shank, C.V., Zholentsand, A. AND Zolotorev, M. (2000). Generation of Femtosecond Pulses of Synchrotron Radiation. Science, 2237.

SundARAM, S.K. AND MAZUR, E. (2002). Inducing and probing non-thermal transitions in semiconductors using femtosecond laser pulses. Nature Materials, Vol 1, 217.
SuWADA, T. (2002). First beam test result of a prototype wire scanner for the KEKB injector linac and BT lines. Proceedings of 11th Symposium on Accelerator Technology and Science, 423 (1997).

SERVICE, R.F. (2002). Battle to become the next generation X-ray source. Science Volume 298, Number 5597, 1356.

Smith, S.L., ET AL. (2004). The Status of the Daresbury Energy Recovery Linac Prototype. Proc. PAC07.

SOKOlOWSKI-TINTEN, K. UND VON DER Linde, D. (2004). Ultrafast phase transitions and lattice dynamics probed using laser-produced x-ray pulses J. Phys. Condens. Matter 16 (2004) R1517R1536.

Smith, S.L. Muratori, B.D. Owen H.L. (2005). Optics designs of ongoing ERL projects. Nucl. Instr. and Meth. A 5.

Sansone, G., Benedetti, E., Calegari, F., Vozzi, C., Avaldi, L., Flammini, R., POletto, L., Villoresi, P., Altucci, C., Velotta, R., Stagira, S., De Silvestri, S., Nisoli M. (2006). Isolated single-cycle attosecond pulses. Science $314,443$.

Tigner, M. (1965). A Possible Apparatus for Electron Clashing Experiments. Nuovo Cimento, Vol. 37. 1228-1231.

TANNEBAUM, P. (1999). Measurement of Small Electron Beam Spots. Annual Review of Nuclear and Particle Science.

Tomov, I.V., Oulianov, D.A., Chen, P. AND RENTZEPIS P.M. (1999). Ultrafast Time-Resolved Transient Structures of Solids and Liquids Studied by Means of X-ray Diffraction and EXAFS. Journal Phys. Chem. B, 103(34) pp 7081.

TÜMmler, J., Janulewicz, K.A., Priebe, G., Nickles, P.V. (2005). 10-Hz grazing-incidence pumped Nilike Mo x-ray laser. Phys. Rev. E, 72 (3), 037401.

UMSTADTER, D. (2003); Relativistic laser plasma interactions. J. Phys. D: Appl. Phys. 36 (8), R151.

VON LAUE, M. (1936). Die äußere Form der Kristalle in ihrem Einfluß auf die Interferenzerscheinungen an Raumgittern. Annalen der Physik, Leipzig 26, 55.

VON DER LINDE, D. (2003). A Picosecond View of Melting. Science Vol. 302 (21), 1345.

WATSON, J.D. AND CRICK, F.H.C. (1953). Implications of the structure of deoxyribonucleic acid. Nature 171, 964-7. 
WARK, J. (1999). X-ray diffraction: Table-top picosecond sources. Nature 398, 284-285.

Xia, B., Li, Z., Kang, K., Huang, W., Huang, G., He, X., DU, Y. AND TANG, C. (2004). Evaluation and simulations of a Thomson scattering $\mathrm{X}$-ray source based on ray tracing methods. Laser and Particle Beams, 22 (3), 355.
ZhAVORONKOV, N., GRITSAI, Y., BARgheER, M., WOERNER, M. AND ElSAESSER, T. (2005). Generation of ultrashort $\mathrm{K}_{\alpha}$ radiation from quasipoint interaction area of femtosecond pulses with thin foils. Appl. Phys. Lett. 86. 